\title{
A new generalization of the Riemann zeta function and its difference equation
}

\author{
Muhammad Aslam Chaudhry ${ }^{\text {* }}$, Asghar Qadir ${ }^{2}$ and Asifa Tassaddiq ${ }^{2}$
}

\author{
* Correspondence: maslam@kfupm. \\ edu.sa \\ ${ }^{1}$ Department of Mathematics and \\ Statistics, King Fahd University of \\ Petroleum and Minerals, Dhahran \\ 31261, Saudi Arabia \\ Full list of author information is \\ available at the end of the article
}

\begin{abstract}
We have introduced a new generalization of the Riemann zeta function. A special case of our generalization converges locally uniformly to the Riemann zeta function in the critical strip. It approximates the trivial and non-trivial zeros of the Riemann zeta function. Some properties of the generalized Riemann zeta function are investigated. The relation between the function and the general Hurwitz zeta function is exploited to deduce new identities.
\end{abstract}

Keywords: Riemann zeta function, Hurwitz zeta function, Polylogarithm function, Extended Fermi-Dirac, Bose-Einstein

\section{Introduction}

The family of zeta functions including Riemann, Hurwitz, Lerch and their generalizations constantly find new applications in different areas of mathematics (number theory, analysis, numerical methods, etc.) and physics (quantum field theory, string theory, cosmology, etc.). A useful generalization of the family is expected to have wide applications in these areas as well. Some extensions of the Fermi-Dirac (FD) and BoseEinstein (BE) functions have been introduced in [1]. The extended Fermi-Dirac (eFD)

$$
\Theta_{\nu}(s ; x):=\frac{1}{\Gamma(s)} \int_{x}^{\infty}(t-x)^{s-1} \frac{e^{-v t}}{e^{t}+1} \mathrm{~d} t \quad(\Re(s)>0 ; x \geq 0 ; \Re(\nu)>-1),
$$

and the extended Bose-Einstein (eBE) functions

$$
\begin{aligned}
\Psi_{\nu}(s ; x):= & \frac{1}{\Gamma(s)} \int_{x}^{\infty}(t-x)^{s-1} \frac{e^{-v t}}{e^{t}-1} \mathrm{~d} t \\
& (\Re(\nu)>-1 ; \Re(s)>1 \text { when } x=0 ; \quad \Re(s)>0 \text { when } x>0),
\end{aligned}
$$

provide a unified approach to the study of the zeta family. These functions proved useful in providing simple and elegant proofs of some known results and yielding new results.

The Hurwitz-Lerch zeta function

$$
\begin{aligned}
& \Phi(z, s, a):=\sum_{n=0}^{\infty} \frac{z^{n}}{(n+a)^{s}} \\
& (s:=\sigma+i \tau, a \neq 0,-1,-2,-3, \ldots ; s \in \mathbb{C} \text { when }|z|<1 ; \sigma>1 \text { when }|z|=1)
\end{aligned}
$$

(C) 2011 Chaudhry et al; licensee Springer. This is an Open Access article distributed under the terms of the Creative Commons Attribution License (http://creativecommons.org/licenses/by/2.0), which permits unrestricted use, distribution, and reproduction in any medium, provided the original work is properly cited.

\section{SpringerOpen ${ }^{\circ}$}


has the integral representation ([[2]2, p. 27, (1.6)(3)])

$$
\begin{aligned}
\Phi(z, s, a) & =\frac{1}{\Gamma(s)} \int_{0}^{\infty} \frac{t^{s-1} e^{-(a-1) t}}{e^{t}-z} \mathrm{~d} t \\
& (\Re(a)>0, \text { and either }-z-\leq 1, z \neq 1, \sigma>0 \text { or } z=1, \sigma>1)
\end{aligned}
$$

If a cut is made from 1 to $\infty$ along the positive real $z$-axis, $\Phi$ is an analytic function of $z$ in the cut $z$-plane provided that $\sigma>0$ and $\Re(a)>0$. A class of functions can be expressed in terms of the function $\Phi$. For example the polylogarithm function

$$
L_{s}(x):=\phi(x, s):=\sum_{n=1}^{\infty} \frac{x^{n}}{n^{s}}=x \Phi(x, s, 1)
$$

Hurwitz's zeta function

$$
\zeta(s, a)=\Phi(1, s, a)
$$

and the Riemann zeta function

$$
\zeta(s)=\zeta(s, 1)=\Phi(1, s, 1),
$$

are special cases of this function. The Hurwitz-Lerch zeta function is related to the above

eFD and eBE functions via

$$
\begin{aligned}
& \Theta_{\nu}(s ; x)=e^{-(v+1) x} \Phi\left(-e^{-x}, s, v+1\right), \\
& \Psi_{\nu}(s ; x)=e^{-(v+1) x} \Phi\left(e^{-x}, s, v+1\right),
\end{aligned}
$$

and shows the extension of the variable $x$ to the complex domain as described in (1.4). The Weyl transform representation of the functions (1.1) and (1.2) leads to new identities for the family of the zeta functions [1].

Here we provide a new generalization of the Riemann zeta function that is also related to the eFD and eBE functions and to the Hurwitz-Lerch zeta function. We study its properties and relations with other special functions. Before defining the new function, it is worth putting the family of zeta functions in perspective for our purpose. Riemann proved that the zeta-function

$$
\zeta(s):=\sum_{n=1}^{\infty} \frac{1}{n^{s}} \quad(s=\sigma+i \tau, \sigma>1),
$$

has a meromorphic continuation to the complex plane, which satisfies the functional equation [[3], p. 13 (2.1.1)]

$$
\zeta(s)=2(2 \pi)^{s-1} \sin \left(\frac{\pi s}{2}\right) \Gamma(1-s) \zeta(1-s)=(\pi)^{s-\frac{1}{2}} \frac{\Gamma\left(\frac{1-s}{2}\right)}{\Gamma\left(\frac{s}{2}\right)} \zeta(1-s) .
$$

From the equation (1.11) it is obvious that $s=-2,-4,6, \ldots$, are simple zeros of the Riemann zeta function. They are called the trivial zeros. It is noted that the simple zero of the sine function on the RHS of (1.11) at $s=0$ is canceled by the simple pole of the zeta function $\zeta(1-s)$ and the simple zeros of the sine function at $s=1,2,3, \ldots$ are canceled by the simple poles of the gamma function $\Gamma(1-s)$ at these points. All 
other zeros of the Riemann zeta function, which are infinitely many as proven by Hardy [4-6], are called the non-trivial zeros, are symmetric about the critical line $\sigma=$ $1 / 2$ in the critical strip $0 \leq \sigma \leq 1$. For the detailed properties of the family of zeta functions we refer to [3-5,7-12]. There have been several generalizations of the Riemann zeta function.

Truesdell [13] studied the properties of the de Jonquière's function or the polylogarithm

$$
\operatorname{Li}_{s}(x)=\phi(x, s)=\sum_{n=1}^{\infty} \frac{x^{n}}{n^{s}}
$$

that generalizes the Riemann zeta function and has the integral representation

$$
\phi(x, s)=\frac{x}{\Gamma(s)} \int_{0}^{\infty} \frac{t^{s-1}}{e^{t}-x} \mathrm{~d} t \quad(|x| \leq 1-\delta, \delta \in(0,1) ; \quad x=1, \sigma>1) .
$$

Note that if $x$ lies anywhere except on the segment of real axis from 1 to $\infty$, where a cut is imposed (1.12) defines an analytic function of $x$ for $\sigma>0$. However (1.12) coincides with the zeta function in $\sigma>1$ for $x=1$ as we have

$$
\operatorname{Li}_{s}(1)=\phi(1, s)=\sum_{n=1}^{\infty} \frac{1}{n^{s}}=\zeta(s) \quad(\sigma>1) .
$$

The Fermi-Dirac (FD) function $\mathfrak{I}_{s-1}(x)$ defined by [[14], p. 20 (25)]

$$
\Im_{s-1}(x):=\frac{1}{\Gamma(s)} \int_{0}^{\infty} \frac{t^{s-1}}{e^{t-x}+1} \mathrm{~d} t \quad(\sigma>0),
$$

and the Bose-Einstein (BE) function defined by [[14], p. 449 (9)]

$$
\beta_{s-1}(x):=\frac{1}{\Gamma(s)} \int_{0}^{\infty} \frac{t^{s-1}}{e^{t-x}-1} \mathrm{~d} t \quad(\sigma>1),
$$

are also related to the zeta family by

$$
\begin{aligned}
& \Im_{s-1}(-x)=-L i_{s}\left(-e^{-x}\right)=-\phi\left(-e^{-x}, s\right)=e^{-x} \Phi\left(-e^{-x}, s, 1\right)=\Theta_{0}(s ; x) \quad(\sigma>0)(1.1 \\
& \beta_{s-1}(-x)=L i_{s}\left(e^{-x}\right)=\phi\left(e^{-x}, s\right)=e^{-x} \Phi\left(e^{-x}, s, 1\right)=\Psi_{0}(s ; x) \quad(\sigma>1)
\end{aligned}
$$

From (1.1), we find that the weighted function

$$
\Gamma(s)\left(1-2^{1-s}\right) \Theta_{v}(s ; 0) \quad(\sigma>0,0 \leq v<1),
$$

converges uniformly to $\gamma(s)\left(1-2^{1-s}\right) \zeta(s)$ as $v \rightarrow 0^{+}$in every sub-strip $0<\sigma_{1} \leq \sigma \leq \sigma_{2}$ $<1$ of the critical strip $0<\sigma<1$. However, for $x=0$ in (1.2) we get

$$
\Psi_{v}(s ; 0):=\frac{1}{\Gamma(s)} \int_{0}^{\infty} t^{s-1} \frac{e^{-v t}}{e^{t}-1} \mathrm{~d} t
$$

which converges to the Riemann zeta function in the region $\sigma \geq \sigma_{1}>1$ as $v \rightarrow 0^{+}$. However, the function (1.20) is not even defined in the critical strip $0<\sigma<1$ as the 
integral is divergent there. So it is desirable to have a generalization of the Riemann and Hurwitz zeta functions in the critical strip, which converges locally uniformly at least. A special case of our new generalization converges to the Riemann zeta function locally uniformly in the critical strip and gives a unified approach, not only to the study of Riemann, Hurwitz, Hurwitz-Lerch zeta functions, but also of the FD and BE functions along with their extensions. An important feature of our approach is the desired simplicity of the proofs using Weyl's fractional transform.

The article is organized as follows. For completeness, in Sect. 2 we state some preliminaries and a general representation formula proved earlier in [1]. In Sect. 3, we define the extended Riemann zeta function and prove its series representation. A connection of the function with the eFD and eBE functions is shown in the next section. In Sect. 5, we prove functional relations of the generalized Riemann zeta function. Some concluding remarks and discussion are given in the last section.

\section{Some preliminaries, Mellin and Weyl's transforms}

The function spaces $\mathrm{H}(\kappa ; \lambda)$ and $\mathrm{H}(\infty ; \lambda)$ are defined as follows (see [1]).

A function $f \in C^{\infty}(0, \infty)$ is said to be a member of $\mathrm{H}(\kappa ; \lambda)$ if:

1. $f(t)$ is integrable on every finite subinterval $[0, T](0<T<\infty)$ of $\mathrm{R}_{0}^{+}:=[0, \infty)$;

2. $f(t)=O\left(t^{-\lambda}\right)\left(t \rightarrow 0^{+}\right)$

3. $f(t)=O\left(t^{-\kappa}\right)(t \rightarrow \infty)$.

Furthermore, if the above relation $f(t)=O\left(t^{-\kappa}\right)(t \rightarrow \infty)$ is satisfied for every exponent $\kappa \in \mathrm{R}_{0}^{+}$, then the function $f(t)$ is said to be in the class $\mathrm{H}(\infty ; \lambda)$. It is noted that $\mathrm{H}(\infty ; \lambda) \subset \mathrm{H}(\kappa ; \lambda)\left(\forall \kappa \in \mathrm{R}_{0}^{+}\right)$. Clearly, we have

$$
f(t)=e^{-b t} \in \mathrm{H}(\infty, 0) \quad(b>0) .
$$

The Mellin transform of $f \in \mathrm{H}(\kappa ; \lambda)$ is defined by (see [[15], p. 83])

$$
f_{M}(s)=M[f(t) ; s]:=\int_{0}^{\infty} f(t) t^{s-1} \mathrm{~d} t \quad(s=\sigma+i \tau, \lambda<\sigma<\kappa) .
$$

The Weyl transform (or Weyl's fractional integral) of order $s$ of $\omega \in \mathrm{H}(\kappa ; 0)$ is defined by (see [[9], Vol. II, p. 181] and [[16], p. 237]),

$$
\begin{aligned}
\Omega(s ; x) & :=W^{-s}[\omega(t)](x):=\frac{1}{\Gamma(s)} M[\omega(t+x) ; s]=\frac{1}{\Gamma(s)} \int_{0}^{\infty} \omega(t+x) t^{s-1} \mathrm{~d} t \\
& =\frac{1}{\Gamma(s)} \int_{x}^{\infty} \omega(t)(t-x)^{s-1} \mathrm{~d} t \quad(s=\sigma+i \tau, \quad 0<\sigma<\kappa, \quad x \geq 0) .
\end{aligned}
$$

For $\sigma \leq 0$, we define the Weyl transform (or Weyl's fractional derivative) of order $s$ of $\omega \in \mathrm{H}(\kappa ; 0)$ as follows (see [[16], p. 241]),

$$
\Omega(s ; x):=W^{-s}[\omega(t)](x):=(-1)^{n} \frac{\mathrm{d}^{n}}{\mathrm{~d} x^{n}}(\Omega(n+s ; x)), \quad(0 \leq n+\sigma<k),
$$


where $n$ is the smallest positive integer greater than or equal to $-\sigma$ provided that $\omega(0)$ is well defined and that

$$
\Omega(0 ; x):=\omega(0) \text {. }
$$

We can rewrite Weyl's fractional derivative (2.4) alternately as

$$
\begin{aligned}
\Omega(-s ; x) & :=W^{s}[\omega(t)](x)=(-1)^{n} \frac{\mathrm{d}^{n}}{\mathrm{~d} x^{n}}\left(W^{-(n-s)}[\omega(t)](x)\right) \\
& =:(-1)^{n} \frac{\mathrm{d}^{n}}{\mathrm{~d} x^{n}}(\Omega(n-s ; x)) \quad(\sigma>0,0 \leq n-\sigma<k),
\end{aligned}
$$

where $n$ is the smallest positive integer greater than or equal to $\sigma$. In particular for $s$ $=n(n=0,1,2,3, \ldots)$ in $(2.6)$, we find that

$$
\Omega(-n ; x):=W^{n}[\omega(t)](x):=(-1)^{n} \frac{\mathrm{d}^{n}}{\mathrm{~d} x^{n}}(\Omega(0 ; x))=(-1)^{n} \frac{\mathrm{d}^{n}}{\mathrm{~d} x^{n}}(\omega(x)) .
$$

Notice that $\left\{W^{s}\right\}(s \in \mathbb{C})$ is a multiplicative group [[16], p. 245] and satisfies

$$
W^{-(\mu+s)}[\omega(t)](x)=W^{-\mu}[\Omega(s ; t)](x)=\Omega(s+\mu ; x] .
$$

The notations $\Re_{s}\{f(t) ; x\}$ and $W_{x+}^{s}[f(t)]$ are also used to represent the Weyl transform (see [[9], Vol. II, p. 181] and [1]). Following the above terminology it was proved in [1] that

$$
\Omega(s ; x)=\sum_{n=0}^{\infty} \frac{(-1)^{n} \Omega(s-n ; 0) x^{n}}{n !} \quad(\omega \in \mathrm{H}(\kappa ; 0), \quad 0 \leq \sigma<k, x \geq 0) .
$$

Note that for the case $\sigma=0,(2.9)$ yields

$$
\begin{gathered}
\Omega(0 ; x)=\sum_{n=0}^{\infty} \frac{(-1)^{n} \Omega(-n ; 0) x^{n}}{n !}=\frac{1}{2 \pi i} \int_{c-i \infty}^{c+i \infty} \Gamma(s) \Omega(s ; 0) x^{-s} \mathrm{~d} s=\frac{1}{2 \pi i} \int_{c-i \infty}^{c+i \infty} \omega_{M}(s) x^{-s} \mathrm{~d} s \\
(\omega \in \mathrm{H}(\kappa ; 0), \quad 0<c<k, x \geq 0),
\end{gathered}
$$

which is Hardy-Ramanujan's master theorem (see [[10], p. 186 (B)]. Some special cases of (2.10) include

$$
\begin{aligned}
\Omega(0 ; x) & =\omega(x):=\left(\frac{1}{e^{x}-1}-\frac{1}{x}\right)=\sum_{n=0}^{\infty} \frac{(-1)^{n} \zeta(-n ; 0) x^{n}}{n !} \\
& =\frac{1}{2 \pi i} \int_{c-i \infty}^{c+i \infty} \Gamma(s) \zeta(s) x^{-s} \mathrm{~d} s=\frac{1}{2 \pi i} \int_{c-i \infty}^{c+i \infty} \omega_{M}(s) x^{-s} \mathrm{~d} s \quad(0<c<1, \quad x \geq 0), \\
Z_{a}(0 ; x) & =z_{a}(x):=\left(\frac{e^{-a x}}{e^{x}-1}-\frac{1}{x}\right)=\sum_{n=0}^{\infty} \frac{(-1)^{n} \zeta(-n ; a) x^{n}}{n !} \\
& =\frac{1}{2 \pi i} \int_{c-i \infty}^{c+i \infty} \Gamma(s) \zeta(s, a) x^{-s} \mathrm{~d} s=\frac{1}{2 \pi i} \int_{c-i \infty}^{c+i \infty} z_{M}(s) x^{-s} \mathrm{~d} s \quad(0<c<1, \quad x \geq 0),
\end{aligned}
$$

which shows that $z_{a}(x) \in H(1 ; 0)(0 \leq a<1)$. Similarly, we have (see [[15], p. 91 (3.3.6)]) 


$$
2 \cos (2 \pi x)=\frac{1}{2 \pi i} \int_{c-i \infty}^{c+i \infty} \frac{\zeta(1-s)}{\zeta(s)} x^{-s} \mathrm{~d} s \quad(0<c<1 / 2, x \geq 0)
$$

which shows that $\cos (2 \pi x) \in H(1 / 2,0)$.

\section{The generalized Riemann zeta function $\Xi_{v}(s ; x)$}

The eFD and the eBE functions defined by (1.1) and (1.2) provide a unified approach to the study of the zeta family. The weighted function $\Gamma(s)\left(1-2^{1-s}\right) \Theta_{v}(s ; 0)$ converges uniformly to $\Gamma(s)\left(1-2^{1-s}\right) \zeta(s)$ as $v \rightarrow 0^{+}$in every sub-strip $0<\sigma_{1} \leq \sigma \leq \sigma_{2}<1$ of the critical strip $0<\sigma<1$. However, the function $\Gamma(s) \Psi_{v}(s ; 0)$ is not even defined in the critical strip as the integral representation (1.1) is divergent in $0<\sigma<1$. It is desirable to have a function that converges uniformly to the Riemann zeta function in some sense and connects the eFD and eBE functions. We assume that $v$ is real and $0 \leq v<1$ in the rest of the article and use analytic continuation [[6], pp. 22-23] to introduce the extended Hurwitz zeta function as follows:

$$
\begin{aligned}
\Xi_{v}(s ; x):= & \frac{1}{\Gamma(s)} \int_{x}^{\infty}(t-x)^{s-1}\left(\frac{1}{e^{t}-1}-\frac{1}{t}\right) e^{-v t} \mathrm{~d} t \\
& (0<\Re(s)<1, \quad x \geq 0, \quad 0 \leq v<1 ; \Re(s)>0, v>0) .
\end{aligned}
$$

For $x=0$ and $v=0$ in (3.1) [[6], p. 22]

$$
\zeta(s) \equiv \Xi_{0}(s ; 0):=\frac{1}{\Gamma(s)} \int_{0}^{\infty} t^{s-1}\left(\frac{1}{e^{t}-1}-\frac{1}{t}\right) \mathrm{d} t \quad(0<\Re(s)<1) .
$$

Theorem 3.1 The generalized Riemann zeta function (3.1) is well defined and the weighted function $\Gamma(s) \Xi_{v}(s ; 0)$ converges uniformly to the weighted Riemann zeta function $\Gamma(s) \zeta(s)$ as $v \rightarrow 0^{+}$in every sub-strip $0<\sigma_{1} \leq \sigma \leq \sigma_{2}<1$ of the critical strip $0<\sigma$ $<1$.

Proof. First we note that

$$
\begin{aligned}
\left|\Gamma(s) \Xi_{v}(s ; 0)\right| & =\left|\int_{0}^{\infty} t^{s-1}\left(\frac{1}{e^{t}-1}-\frac{1}{t}\right) e^{-v t} \mathrm{~d} t\right| \leq\left|\int_{0}^{\infty} t^{\sigma-1}\left(\frac{1}{t}-\frac{1}{e^{t}-1}\right) e^{-\nu t} \mathrm{~d} t\right| \\
& \leq \int_{0}^{\infty} t^{\sigma-1}\left(\frac{1}{t}-\frac{1}{e^{t}-1}\right) \mathrm{d} t=-\Gamma(\sigma) \Xi_{0}(\sigma)=-\Gamma(\sigma) \zeta(\sigma),
\end{aligned}
$$

which shows that the generalized Riemann zeta function (3.1) is well defined. Second, that the difference integral representation (as $1-e^{-v t} \leq 1,0 \leq v<1,0 \leq t<\infty$ ),

$$
\begin{aligned}
\left|\Gamma(s)\left(\Xi_{v}(s ; 0)-\zeta(s)\right)\right|= & \left|\int_{0}^{\infty} t^{s-1}\left(\frac{1}{e^{t}-1}-\frac{1}{t}\right)\left(e^{-v t}-1\right) \mathrm{d} t\right| \leq \int_{0}^{\infty} t^{\sigma-1}\left(\frac{1}{t}-\frac{1}{e^{t}-1}\right)\left(1-e^{-v t}\right) \mathrm{d} t \\
\leq & \int_{0}^{\infty} t^{\sigma-1}\left(\frac{1}{t}-\frac{1}{e^{t}-1}\right) \mathrm{d} t=-\Gamma(\sigma) \Xi_{0}(\sigma)=-\Gamma(\sigma) \zeta(\sigma) \\
& \left(0 \leq v<1, \quad 0<\sigma_{1} \leq \sigma \leq \sigma_{2}<1\right),
\end{aligned}
$$

is absolutely convergent shows that the limit as $v \rightarrow 0^{+}$and the integral in (3.4) are reversible. Letting $v \rightarrow 0^{+}$in (3.4) we find that the convergence 


$$
\left|\Gamma(s)\left(\Xi_{v}(s ; 0)-\zeta(s)\right)\right| \rightarrow 0 \quad\left(v \rightarrow 0^{+}, 0<\sigma_{1} \leq \sigma \leq \sigma_{2}<1\right),
$$

is uniform.

Theorem 3.2 (Connection with the Hurwitz-zeta function)

$$
\begin{aligned}
\Xi_{v}(s ; 0)=\zeta(s, v+1)-\frac{\Gamma(s-1)}{\Gamma(s)} v^{1-s} & =\zeta(s, v+1)-\frac{1}{s-1} v^{1-s} \\
& (0<v<1, \quad \sigma>0, \quad v=0, \quad 0<\sigma<1) .
\end{aligned}
$$

Proof. We assume that $0<v<1$ and $\sigma>1$. In this case, from (3.1)

$$
\begin{aligned}
\Xi_{v}(s ; 0):= & \frac{1}{\Gamma(s)} \int_{0}^{\infty} t^{s-1}\left(\frac{1}{e^{t}-1}-\frac{1}{t}\right) e^{-v t} \mathrm{~d} t=\frac{1}{\Gamma(s)} \int_{0}^{\infty} t^{s-1} \frac{e^{-v t}}{e^{t}-1} \mathrm{~d} t-\frac{1}{\Gamma(s)} \int_{0}^{\infty} t^{s-2} e^{-v t} \mathrm{~d} t \\
= & \zeta(s, v+1)-\frac{\Gamma(s-1)}{\Gamma(s)} v^{1-s}=\zeta(s, v+1)-\frac{\Gamma(s-1)}{(s-1) \Gamma(s-1)} v^{1-s}=\zeta(s, v+1)-\frac{1}{s-1} v^{1-s} \\
& (0<v<1, \sigma>1) .
\end{aligned}
$$

Note that the RHS in (3.7) remains well defined for $0<\sigma<1$ and $0<v<1$. Moreover, for $v=0$, we have the well known integral representation (3.2) (see [[6], p. 22]) for $0<\sigma<1$. Hence the proof.

Remark 3.3 The representation (3.6) of the generalized Riemann zeta function shows that the function is meromorphic. For $v \in(0,1)$ the function has a removable singularity at $s=1$ as the residue of the function is zero. However, for $v=0$ the function has a simple pole at $s=1$ with residue 1 . We can rewrite (3.6) as

$$
\Xi_{v}(s ; 0)=\frac{1}{s-1}\left[(s-1) \zeta(s, v+1)-v^{1-s}\right] \quad(0<v<1 ; v=0, \quad 0<\sigma<1) .
$$

Putting $s=-n$ and using [[7], p. 264]

$$
\zeta(-n, a)=-\frac{B_{n+1}(a)}{n+1} \quad(n=0,1,2, \ldots)
$$

we find that the function is related to the Bernoulli's polynomials via (see [[7], p. 264, (17)])

$$
\Xi_{v}(-n, 0)=\frac{v^{n+1}-B_{n+1}(v+1)}{n+1} \quad(0<v<1, n=0,1,2,3, \ldots) .
$$

Using the relations (see [[11], pp. 26-28])

$$
\begin{aligned}
& B_{2 n+1}(v+1)=B_{2 n+1}(v)+(2 n+1) v^{2 n}, \\
& B_{2 n+1}(v)=\sum_{k=0}^{2 n+1}\left(\begin{array}{l}
2 n+1 \\
k
\end{array}\right) B_{k} v^{2 n+1-k}, \\
& B_{2 n+1}(0)=: B_{2 n+1}=(2 n+1) \zeta(-2 n)=0, \\
& B_{2 n}(0)=: B_{2 n}=-2 n \zeta(1-2 n) \quad(n=1,2,3, \ldots),
\end{aligned}
$$

and

$$
B_{2 n}(0)=: B_{2 n}=-2 n \zeta(1-2 n) \sim(-1)^{n+1} \frac{(4 n) !}{(2 \pi)^{2 n}}\left(1+2^{-2 n}\right) \quad(n=3,4,5, \ldots)(3
$$


we obtain the closed form

$$
\begin{aligned}
\Xi_{v}(-2 n, 0) & =\frac{v^{2 n+1}-B_{2 n+1}(v+1)}{2 n+1}=\frac{v^{2 n+1}-\left(B_{2 n+1}(v)+(2 n+1) v^{2 n}\right)}{2 n+1} \\
& =\frac{v^{2 n+1}-\left\{\begin{array}{l}
2 n+1 \\
k=0
\end{array}\left(\begin{array}{l}
2 n+1 \\
k
\end{array}\right) B_{k} v^{2 n+1-k}+(2 n+1) v^{2 n}\right\}}{2 n+1} \quad(n=1,2,3, \ldots),
\end{aligned}
$$

which shows that

$$
\Xi_{v}(-2 n, 0)=-B_{2 n} v+O\left(v^{3}\right)=2 n \zeta(1-2 n) v+O\left(v^{3}\right) \quad\left(v \rightarrow 0^{+}, n=1,2,3, \ldots\right)(3
$$

Thus the generalized Riemann zeta function approximates the trivial zeros $(s=-2$, $-4,-6, \ldots)$ of the Riemann zeta function as $v \rightarrow 0^{+}$. The relation (3.17) gives the rate at which these zeros are approached. One needs to see if all the zeros can be approximated uniformly. Since $|2 n \zeta(1-2 n)| \rightarrow \infty$ as $n \rightarrow \infty$, by setting

$$
v_{n, k}=\frac{2^{-k}}{|2 n \zeta(1-2 n)|} \quad(n=1,2,3, \ldots),
$$

we have

$$
\sup _{1 \leq n<\infty}\left|\Xi_{v_{n, k}}(-2 n, 0)\right|=\circ(1) \quad(k \rightarrow \infty) .
$$

which shows that all the non-trivial zeros can, indeed, be approximated uniformly.

Remark 3.4 It is worth visualizing the behavior of the function near $v=0$ for large $n$ more generally. Though $\Xi_{v}(-n, 0)$ is a function of one continuous and one discrete variable, conceive it as if it were a sheet over the strip $v \in(0,1), n \in(0, \infty)$ in the $(v$, $n)$-plane. At every $n$ the sheet approaches the $n$-axis arbitrarily closely, but it does not do so for all $n$, since the sheet rises increasingly more sharply for larger values of $n$. The asymptotic formula for $\zeta(1-2 n)$ (see [[11], pp. 26-28]) can be used in conjunction with Stirlings formula to give the coefficient of $v$ (for small $v$ )

$$
B_{2 n}(0) \sim(-1)^{n+1} \frac{(4 n) !}{(2 \pi)^{2 n}}\left(1+2^{-2 n}\right) \sim(-1)^{n+1} \sqrt{18 \pi n}\left(8 n^{2} / \pi e^{2}\right)^{2 n} .
$$

The function of the discrete variable can be thought of as the parts of the sheet lying over the grid lines of the integer values of $n$. The sequence where the curve intersects the grid lines gives a path. The non-trivial zeros are then clearly uniformly approximated by paths approaching $v=0$ lying between $v=1 /\left|2 n \zeta\left(1-2_{n}\right)\right|$ and the $n$-axis.

\section{Connection with the eFD and eBE integral functions}

Theorem 4.1 The generalized Riemann zeta function is related to the eBE integral functions and the incomplete gamma function via

$$
\begin{array}{r}
\Psi_{v}(s ; x)=\Xi_{v}(s ; x)+\Gamma(1-s, v x) x^{s-1} \\
(\sigma>0, \quad v>0, \quad x>0 ; \quad v=0, \quad 0<\sigma<1, x \geq 0) .
\end{array}
$$

Proof. We have the identity

$$
\frac{e^{-v t}}{e^{t}-1}=\left(\frac{1}{e^{t}-1}-\frac{1}{t}\right) e^{-v t}+\frac{e^{-v t}}{t} .
$$


By taking the Weyl's transform of both sides in (4.2) we obtain

$$
\Psi_{\nu}(s ; x)=\Xi_{\nu}(s ; x)+W^{-s}\left[\frac{e^{-v t}}{t}\right](x) .
$$

However, we have (see [[9], pp. 255, 266])

$$
W^{-s}\left[\frac{e^{-v t}}{t}\right](x)=x^{s-1} e^{-v x} \psi(s, s ; \nu x)=x^{s-1} \Gamma(1-s, v x) \quad(\sigma>0, v>0, x>0) .
$$

From (4.3) and (4.4) we arrive at (4.1).

\section{Corollary 4.2}

$$
\beta_{s-1}(-x)=\Xi_{0}(s ; x)+\Gamma(1-s) x^{s-1} \quad(0<\sigma<1, x>0)
$$

Proof. This follows from (4.1) when we take $v=0$ and use (1.20).

\section{Theorem 4.3}

$$
\begin{aligned}
\Xi_{\nu}(s ; x) & =\sum_{n=0}^{\infty} \frac{(-1)^{n} \Xi_{\nu}(s-n: 0) x^{n}}{n !} \\
& (\sigma>0, \quad v>0, \quad x>0 ; \quad v=0, \quad 0<\sigma<1, \quad 0<x<2 \pi) .
\end{aligned}
$$

Proof. First we note that

$$
\Xi_{\nu}(0, x):=\left(\frac{1}{e^{x}-1}-\frac{1}{x}\right) e^{-v x} \in H(\infty ; 0) .
$$

Therefore, following the general expansion result (2.9), we arrive at (4.6).

Remark 4.4 A very interesting special case of (4.6) arises when $v=s=0$. In this case we have the well-known result proved by Hardy and Littlewood [5]

$$
\Xi_{0}(0 ; x)=\frac{1}{e^{x}-1}-\frac{1}{x}=\sum_{n=0}^{\infty} \frac{(-1)^{n} \zeta(-n) x^{n}}{n !} \quad(0<x<2 \pi) .
$$

Equations (4.5) and (4.6) lead to the useful representation

$$
\beta_{s-1}(-x)=\Gamma(1-s) x^{s-1}+\sum_{n=0}^{\infty} \frac{(-1)^{n} \zeta(s-n: 0) x^{n}}{n !} \quad(0<\sigma<1, \quad 0<x<2 \pi) .
$$

Theorem 4.5 The generalized Riemann zeta and the eFD integral functions are related by

$$
\begin{aligned}
& 2^{1-s} \Xi_{v}(s ; 2 x)=\Xi_{2 v}(s ; x)-\Theta_{2 v}(s, x) \\
&(\sigma>0, \quad v>0, \quad x>0 ; \quad v=0, \quad 0<\sigma<1, \quad x \geq 0) .
\end{aligned}
$$

Proof. We have the identity

$$
2\left(\frac{e^{-2 v t}}{e^{2 t}-1}-\frac{e^{-2 v t}}{2 t}\right)=\left(\frac{1}{e^{t}-1}-\frac{1}{t}\right) e^{-2 v t}-\frac{e^{-2 v t}}{e^{t}+1} .
$$


Taking the Weyl transform of both sides in (4.11), we find that

$$
\begin{aligned}
& 2 W^{-s}\left[\frac{e^{-2 v t}}{e^{2 t}-1}-\frac{e^{-2 v t}}{2 t}\right](x) \\
& \quad=W^{-s}\left[\frac{e^{-2 v t}}{e^{t}-1}-\frac{e^{-2 v t}}{t}\right](x)-W^{-s}\left[\frac{e^{-2 v t}}{e^{t}+1}\right](x)=\Xi_{2 v}(s ; x)-\Theta_{2 v}(s, x) .
\end{aligned}
$$

However, we have

$$
2 W^{-s}\left[\frac{e^{-2 v t}}{e^{2 t}-1}-\frac{e^{-2 v t}}{2 t}\right](x)=\frac{2}{\Gamma(s)} \int_{x}^{\infty}(t-x)^{s-1}\left(\frac{1}{e^{2 t}-1}-\frac{1}{2 t}\right) e^{-2 v t} \mathrm{~d} t .
$$

The substitution $t=\tau / 2$ in (4.13) leads to

$$
\begin{gathered}
2 W^{-s}\left[\frac{e^{-2 v t}}{e^{2 t}-1}-\frac{e^{-2 v t}}{2 t}\right](x)=\frac{1}{\Gamma(s)} \int_{2 x}^{\infty}(\tau / 2-x)^{s-1}\left(\frac{1}{e^{\tau}-1}-\frac{1}{\tau}\right) e^{-v \tau} \mathrm{d} \tau \\
=\frac{2^{1-s}}{\Gamma(s)} \int_{2 x}^{\infty}(\tau-2 x)^{s-1}\left(\frac{1}{e^{\tau}-1}-\frac{1}{\tau}\right) e^{-\nu \tau} \mathrm{d} \tau=2^{1-s} \Xi_{\nu}(s ; 2 x)
\end{gathered}
$$

From (4.12), (4.13), and (4.14) we arrive at (4.10).

Remark 4.6 It is useful to write (4.10) in the form

$$
\begin{aligned}
\Theta_{2 v}(s, x) & =\Xi_{2 v}(s ; x)-2^{1-s} \Xi_{v}(s ; 2 x) \\
& (\sigma>0, \quad v>0, \quad x>0 ; \quad v=0,0<\sigma<1, \quad x \geq 0) .
\end{aligned}
$$

Putting $v=x=0$ in (4.15) we find the classical integral representation

$$
\Theta_{0}(s, 0)=\left(1-2^{1-s}\right) \zeta(s)=\frac{1}{\Gamma(s)} \int_{0}^{\infty} \frac{t^{s-1}}{e^{t}+1} \mathrm{~d} t \quad(\sigma>0)
$$

for the weighted Riemann zeta function. Note that the simple pole of the zeta function at $s=1$ is cancelled by the (simple) zero of the factor $1-2^{1-s}$ such that the product $\Theta_{0}(s, 0)=\left(1-2^{1-s}\right) \zeta(s)$ remains well defined in the sense of the Riemann removable singularity theorem. Moreover using the relations (1.8) and (1.9) we can rewrite (4.10) in terms of the Hurwitz-Lerch zeta function as

$$
\begin{aligned}
\Xi_{2 v}(s ; x)-2^{1-s} \Xi_{v}(s ; 2 x) & =e^{-(2 v+1) x} \Phi\left(-e^{-x}, s, 2 v+1\right) \\
& (\sigma>0, v>0, x>0 ; \quad v=0,0<\sigma<1, x \geq 0) .
\end{aligned}
$$

This can be extended to a function of the complex variable $z$ as given in (1.4).

Corollary 4.7 (Connection with the FD functions)

$$
\Xi(s ; x)-2^{1-s} \Xi(s ; 2 x)=\Im_{s-1}(-x) \quad(0<\sigma<1) .
$$

Proof. This follows from (1.17) and (1.18) and from (4.10) when we put $v=0$.

\section{Difference equation for the generalized Riemann zeta function}

Functional relations arising from difference equations are useful for the study of special functions. For example, the Bernoulli polynomials satisfy the difference equation ([[7], p. 265 (18)]) 


$$
B_{n}(x+1)-B_{n}(x)=n x^{n-1} \quad(n \geq 1) .
$$

Do the generalized Riemann-zeta functions also satisfy such relations? The next theorem gives the answer to this question.

Theorem 5.1 The generalized Riemann zeta function $\Xi_{v}(s ; x)$ satisfies the difference equation

$$
\begin{aligned}
& \Xi_{v}(s ; x)-\Xi_{v+1}(s ; x) \\
& =(v+1)^{-s} e^{-(v+1) x}-x^{s-1}(\Gamma(1-s, v x)-\Gamma(1-s,(v+1) x)) \\
& \quad(x, v>0, \sigma>0 ; \quad x>0, v=0,0<\sigma<1) .
\end{aligned}
$$

Proof. We have the identity

$$
\frac{e^{-v t}}{e^{t}-1}-\frac{e^{-v t}}{t}-\frac{e^{-(v+1) t}}{e^{t}-1}+\frac{e^{-(v+1) t}}{t}=e^{-(v+1) t}-\frac{e^{-v t}}{t}+\frac{e^{-(v+1) t}}{t} \quad(v \geq 0) .
$$

Applying the Weyl transform on both sides in (5.3) we obtain

$$
\begin{aligned}
& W^{-s}\left[\frac{e^{-v t}}{e^{t}-1}-\frac{e^{-v t}}{t}\right](x)-W^{-s}\left[\frac{e^{-(v+1) t}}{e^{t}-1}-\frac{e^{-(v+1) t}}{t}\right](x) \\
& =W^{-s}\left[e^{-(v+1) t}\right](x)-W^{-s}\left[\frac{e^{-v t}}{t}\right](x)+W^{-s}\left[\frac{e^{-(v+1) t}}{t}\right](x) . .
\end{aligned}
$$

However (see [[9], Vol. II, p. 202]),

$$
W^{-s}\left[e^{-a t}\right](x)=a^{-s} e^{-a x},
$$

and (see [[2], p. $262(6.9 .2)(21)])$

$$
W^{-s}\left[\frac{1}{t} e^{-a t}\right](x)=x^{s-1} e^{-a x} \psi(s, s ; a x)=x^{s-1} \Gamma(1-s, a x) .
$$

From (5.4), (5.5), and (5.6) we arrive at (5.2).

\section{Concluding remarks and discussion}

According to Bombieri (see [[17], (2)]), the formula

$$
\zeta(s)+1-\frac{1}{s-1}=\frac{1}{\Gamma(s)} \int_{0}^{\infty}\left(\frac{1}{e^{t}-1}-\frac{1}{t}\right) e^{-t} \mathrm{~d} t
$$

was proved by Tchebychev, from which he deduced that $(s-1) \zeta(s)$ has limit one as $s$ $\rightarrow 1$. He used the above formula in his first memoir to prove the asymptotic formula for the number of primes less than a given number. Putting $v=1$ in (3.6) we have

$$
\Xi_{1}(s ; 0)=\zeta(s, 2)-\frac{1}{s-1}=\zeta(s)+1-\frac{1}{s-1}=\frac{1}{\Gamma(s)} \int_{0}^{\infty}\left(\frac{1}{e^{t}-1}-\frac{1}{t}\right) e^{-t} \mathrm{~d} t,
$$

which is exactly Tchebychev's formula. This shows that a very special case of our new generalized Riemann zeta function appeared earlier in the work of Tchebychev in the study of the Riemann zeta function and the location of the non-trivial zeros. However, the general case of the function and its relation with the zeta family does not seem to have been realized so far. We studied the properties and functional relations of the new function. It achieves the desired simplification of the cumbersome proofs of elegant properties of the Hurwitz-Lerch zeta function. This simplification can be 
expected to lead to other results that may have remained unproven due to the complexity of their proofs.

It was seen that the generalized Riemann zeta function has simple connections with the recently defined eBE and eFD functions, which have also found another use in Physics. For certain problems arising in condensed matter theory quasi-particles that are neither fermions nor bosons had been proposed. They had been called "anyons" (see references in [18] for the literature on these particles). The eBE and eFD functions had been put forward as possible candidates for the anyon function as they interpolate very naturally between the $\mathrm{BE}$ and FD functions. The above connections enable us to obtain an asymptotic expansion of the function in the critical strip. It turns out that the new function is also related to the Bernoulli polynomials via (3.12) and approximates the non-trivial zeros of the Riemann zeta function as well.

\section{Abbreviations}

BE: Bose-Einstein; eFD: extended Fermi-Dirac; FD: Fermi-Dirac.

\section{Acknowledgements}

Two of the authors (MAC and $A Q$ ) are grateful to the King Fahd University of Petroleum and Minerals for providing excellent research facilities. AT acknowledges her indebtedness to the Higher Education Commission of Pakistan for the Indigenous PhD Fellowship.

\section{Author details \\ 'Department of Mathematics and Statistics, King Fahd University of Petroleum and Minerals, Dhahran 31261, Saudi Arabia ${ }^{2}$ Center for Advanced Mathematics and Physics, National University of Science and Technology H-12, Islamabad, Pakistan}

\section{Authors' contributions}

All authors contributed equally to the manuscript and read and approved the final draft.

\section{Competing interests}

The authors declare that they have no competing interests.

Received: 1 January 2011 Accepted: 30 June 2011 Published: 30 June 2011

\section{References}

1. Srivastava HM, Chaudhry MA, Qadir A, Tassaddiq A: Some extensions of the Fermi-Dirac and Bose-Einstein functions with applications to the family of zeta functions. Russian J. Math. Phys 2011, 18:107-121.

2. Erdélyi A, Mangus W, Oberhettinger F, Tricomi FG: Higher Transcendental Functions. McGraw-Hill Book Company, New York, Toronto and London; 19531.

3. Temme NM: Special Functions: An Introduction to Classical Functions of Mathematical Physics. Wiley, New York, Chichester, Brisbane, Toronto; 1996.

4. Edwards HM: Riemann Zeta Function. Academic Press, New York; 1974

5. Hardy GH, Littlewood JE: Contributions to the theory of the Riemann zeta function and the theory of the distribution of primes. Acta Math 1918, 41:119-196.

6. Titchmarsh EC: The Theory of the Riemann Zeta Function. Clarendon (Oxford University) Press, Oxford, London, New York; 1951.

7. Apostol TM: Introduction to Analytic Number Theory. Springer-Verlag, Berlin, New York, Heidelberg; 1976.

8. Chaudhry MA, Zubair SM: On a Class of Incomplete Gamma Functions with Applications. Chapman and Hall (CRC Press), Boca Raton; 2001.

9. Magnus W, Oberhettinger F, Tricomi FG: Tables of Integral Transforms, Vols I and II. McGraw-Hill Book Company, New York, Toronto and London; 1954.

10. Hardy GH: Ramanujan: Twelve Lectures on Subjects Suggested by His Life and Work. Chelsea Publishing Company, New York; 1959.

11. Magnus W, Oberhettinger F, Soni RP: Formulas and Theorems for the Special Functions of Mathematical Physics. Springer-Verlag, Berlin, New York, Heidelberg; 1966.

12. Srivastava HM: Some formulas for the Bernoulli and Euler polynomials at rational arguments. Math. Proc. Cambridge Philos. Soc 2000, 129:77-84.

13. Truesdell C: On a function which occurs in the theory of the structure of polymers. Ann. Math 1945, 46:144-157.

14. Dingle RB: Asymptotic Expansions: Their Derivation and Interpretation. Academic Press, London and New York; 1973.

15. Paris RB, Kaminski D: Asymptotics and Mellin-Barnes Integrals. Cambridge University Press, Cambridge, London, New York; 2001.

16. Miller KS, Ross B: An Introduction to the Fractional Calculus and Fractional Differential Equations. Wiley, New York, Chichester, Brisbane, Toronto; 1993. 
17. Bombieri E: Problem of the Mellennium: The Riemann hypothesis.[http://www.claymath.org/millennium/ Riemann_Hypothesis/].

18. Chaudhry MA, Iqbal A, Qadir A: A representation for the anyon integral function. 2005, [arXiv:math-ph/0504081].

doi:10.1186/1687-1847-2011-20

Cite this article as: Chaudhry et al: A new generalization of the Riemann zeta function and its difference equation. Advances in Difference Equations 2011 2011:20.

Submit your manuscript to a SpringerOpen ${ }^{\odot}$ journal and benefit from:

- Convenient online submission

- Rigorous peer review

- Immediate publication on acceptance

- Open access: articles freely available online

- High visibility within the field

- Retaining the copyright to your article

Submit your next manuscript at $\boldsymbol{\nabla}$ springeropen.com 